\title{
A produção intelectual em Saúde Coletiva no âmbito do Estado do Rio de Janeiro: uma trajetória de 1960 a 2007
}

I 1 George Edward Machado Kornis, ${ }^{2}$ Leila Senna Maia,

${ }^{3}$ Renata Ferraiolo Peixoto Fortuna I

Resumo: Este artigo tem como foco a produção intelectual em Saúde Coletiva nos níveis de mestrado e doutorado cujo tema está no âmbito do Estado do Rio de Janeiro. A pesquisa considerou o período compreendido entre 1960 e 15 de julho de 2007. No subperíodo entre 1960 e 1990, foi encontrado apenas um trabalho no campo da Saúde Coletiva cujo tema foi o Estado do Rio de Janeiro. No subperíodo compreendido entre 1991 e 15 de julho de 2007, foram encontrados 48 trabalhos no campo da Saúde Coletiva referentes a esse estado. A produção foi classificada inicialmente em oito áreas temáticas; Políticas; Epidemiologia; Saúde e Sociedade; Recursos Humanos; Formação Profissional; Modelos de Atenção e de Gestão; Sistemas de Informação e História da Saúde Pública. A área temática Políticas, que lidera a produção, concentrando 22 trabalhos, foi então dividida em duas subcategorias: Políticas do SUS, com 20\%, e Políticas Específicas, com $25 \%$. A área temática Epidemiologia, na qual foram encontrados 11 trabalhos (23\%), representa outro polo de concentração importante. A produção intelectual total esteve concentrada tanto em dissertaçôes de mestrado (78\%) como institucionalmente: a FIOCRUZ lidera a produção de dissertações de mestrado, enquanto o IMS/UERJ lidera a de teses de doutoramento. A despeito da importância do Estado do Rio de Janeiro no Brasil, a pesquisa conclui que a produção intelectual no campo da Saúde Coletiva referente a esse estado é baixa e concentrada em poucas áreas temáticas.

> Palavras-chave: Produção intelectual; Saúde Coletiva; Estado do Rio de Janeiro.
1 Professor Adjunto no

Departamento de Planejamento e Administração de Saúde do IMS-UERJ; Economista; Mestre e Doutor em Economia pelo Instituto de Economia da Universidade Federal do Rio de Janeiro. Endereço eletrônico: kornis@ims.uerj.br.

${ }^{2}$ Cirurgiã-dentista; Especialista em Odontopediatria pela Policlínica Geral do Rio de Janeiro; Especialista em Programa de Saúde da Família pelo Consórcio MS/EURJ-"PSFRJ"; Mestre e doutoranda em Saúde Coletiva pelo IMS-UERJ, sob orientação do prof. George Kornis. Endereço eletrônico: senna.maia@ims.uerj.br.

${ }^{3}$ Cirurgiã-dentista; Especialista em Endodontia pela Faculdade de Odontologia de Campos; Mestre e Doutoranda em Saúde Coletiva pelo IMS-UERJ, sob a orientação do prof. George Kornis. Endereço eletrônico: renataferraiolo@hotmail.com.

Recebido em: 27/07/2009. Aprovado em: 30/04/2010. 


\section{Apresentação}

$\mathrm{O}$ interesse em aprofundar os conhecimentos acerca das particularidades que compóem o arranjo institucional da saúde pública no âmbito do Estado Rio de Janeiro levou um grupo composto por professor e discentes do Instituto de Medicina Social da Universidade do Estado do Rio de Janeiro (IMS-UERJ) a assumir, em junho de 2007, a seguinte tarefa: realizar um levantamento da produção acadêmica expressa em dissertações de mestrado e teses de doutoramento produzidas e indexadas de 1960 até 15 de julho de 2007 com temas voltados para a Saúde Coletiva no âmbito deste estado.

$\mathrm{O}$ referido grupo de pesquisadores publicou, em 2006, um trabalho intitulado "A produção intelectual em saúde bucal na perspectiva coletiva: uma análise da experiência brasileira de 1990 a 2006". Esse artigo pioneiro pesquisou exclusivamente trabalhos voltados para a saúde bucal na perspectiva coletiva e teve abrangência nacional. Durante sua execução, foram selecionadas as bases de dados e desenvolvidos os recursos metodológicos que servem também de matriz para o presente artigo e servirão de referência para futuras publicações a serem realizadas nos mesmos moldes.

$\mathrm{O}$ processo de desenvolvimento do artigo publicado em 2006 permitiu identificar a baixa densidade de trabalhos voltados para ampliar o conhecimento a respeito da Saúde Coletiva no Estado do Rio de Janeiro. Neste sentido, o presente artigo representa um esforço de pesquisa orientado para criar conhecimento a respeito da produção científica em Saúde Coletiva nos níveis mestrado e doutorado no âmbito desse estado.

Os objetivos deste trabalho foram: identificar nichos temáticos no conjunto da produção; estimular a pesquisa, de modo a preencher as lacunas encontradas na produção bibliográfica e dar continuidade a uma sequência de publicações acerca da produção científica em Saúde Coletiva no Brasil, a partir de uma matriz metodológica comum e sob diferentes recortes. Assim, o presente artigo está estruturado da seguinte maneira: na Introdução, é feito um relato dos principais motivos que levaram os autores a investigar o tema. Ademais, é feita uma breve descrição das principais características do Estado do Rio de Janeiro. No tocante à Metodologia, são apresentados os procedimentos de trabalho adotados no âmbito da pesquisa. Na seção Resultados, são expostos os principais dados coletados e sistematizados no curso do trabalho. A Discussão faz uma análise dos dados frente 
aos principais argumentos desenvolvidos no processo da pesquisa e, finalmente, no último segmento, Considerações finais, é feito um breve exercício de análise prospectiva a partir dos resultados obtidos na presente investigação.

\section{Introdução}

O ponto de partida do presente artigo foi o trabalho seminal organizado pelas professoras Lícia do Prado Valladares e Maria Josefina G. Sant’Anna (VALLADARES; SANT'ANNA, 1992), intitulado O Rio de Janeiro em Teses: catálogo bibliográfico 1960-1990. Essa publicação identificou um total de 265 trabalhos, entre dissertações de mestrado e teses de doutoramento. ${ }^{1}$ Esses trabalhos tiveram como tema, de forma direta ou indireta, o Rio de Janeiro, considerando-se tanto o município como o estado. Deste montante, apenas oito trabalhos (3\%) foram desenvolvidos de modo a contemplar exclusivamente o Estado do Rio de Janeiro. Contudo, deste reduzido volume de trabalhos, apenas um está referido ao campo da Saúde Coletiva: a dissertação de mestrado intitulada Trabalho médico no Rio de Janeiro, de Jane de Araújo Oliveira (OLIVEIRA, 1980).

Esta constatação nos levou a investigar o comportamento dessa produção no período subsequente ao da publicação supracitada, ou seja, a partir de 1991. Desta forma, o corte temporal adotado pelo presente artigo tem início em 1960 e se estende até 15 de julho de 2007. No tocante ao subperíodo compreendido entre 1991 e 2007, foram empregadas as principais bases de dados descritas no item Metodologia do presente trabalho. Neste último período, foram encontrados 48 trabalhos que tiveram como tema o Estado do Rio de Janeiro. Assim, o contraste existente entre a produção dos dois períodos pesquisados é evidente. Contudo, o total da produção (49) considerada na presente pesquisa é ainda muito baixo.

A baixa densidade de trabalhos que tiveram como tema o Estado do Rio de Janeiro é incompatível com a complexidade do arranjo institucional que dá sustentação à atenção à saúde pública no âmbito desse estado. Os fundamentos históricos dessa complexidade remontam ao longo período no qual a cidade do Rio de Janeiro foi a capital do país, desempenhando também importantes funçōes econômicas e culturais. Ademais, a criação de Brasília (1960) e a consequente perda do status de capital, somadas ao processo da fusão (1975) do extinto Estado da Guanabara (hoje município do Rio de Janeiro) com o Estado do Rio de Janeiro 
acabaram por imprimir características peculiares no campo da saúde pública na formação da nova unidade federativa (PARADA, 1997 e 2002; MAIA, 2008).

Enquanto herança destes processos históricos, a cidade do Rio de Janeiro acumula hoje a maior rede de serviços federais de saúde pública do país, imprimindo características singulares ao sistema de saúde no âmbito estadual (LIMA, 2001; MAIA, 2008).

Sob outro ponto de análise, o Estado do Rio de Janeiro é a unidade federativa com maior grau de urbanização (96\%) do país (SANTOS, 2003), tendo como característica a forte concentração da sua população residindo na região metropolitana (73,68\%). O outro lado desta concentração está expresso no forte desequilíbrio econômico e social diante das diversas regiōes que compõem o interior do estado (MAIA, 2008).

O impacto dessas desigualdades estruturais sobre as condições de assistência à saúde no Estado do Rio de Janeiro não é desprezível. Este impacto deveria, a princípio, estimular uma maior produção acadêmica acerca dessa unidade da federação no campo da saúde coletiva. Ademais, considerando a diretriz da descentralização da política nacional de saúde ratificada pelo Pacto Pela Saúde de 2006, é imperativo para o êxito do SUS que se amplie o conhecimento acerca das realidades sanitárias das unidades da federação, assim como de seus respectivos municípios.

Nesta perspectiva, o presente trabalho, ao buscar mensurar a produção acadêmica expressa em dissertaçóes de mestrado e teses de doutoramento em Saúde Coletiva no âmbito do Estado do Rio de Janeiro, pretende ser um subsídio para o desenvolvimento da pesquisa acadêmica orientada para e o planejamento/ gestão das ações em saúde nessa unidade da federação.

\section{Metodologia}

A presente pesquisa teve como foco as dissertações de mestrado e as teses de doutoramento voltadas para a Saúde Coletiva no âmbito do Estado do Rio de Janeiro no período de 1960 até 15 de julho de 2007. A pesquisa realizada para o período compreendido entre 1960 e 1990 concentrou-se no catálogo bibliográfico O Rio de Janeiro em Teses de 1960 a 1990 (VALLADARES; SANT'ANNA, 1992). A pesquisa relativa ao período subsequente (1991 a 15 de julho de 2007) seguiu o mesmo desenho metodológico de outro trabalho realizado pelo mesmo 
grupo de pesquisadores (KORNIS; MAIA; FORTUNA, 2006). Essa opção metodológica teve o objetivo de tornar possível alguma comparabilidade entre dos dois estudos. Foram utilizadas as seguintes bases de dados: ${ }^{2}$

- Portal CAPES de teses e dissertaçōes (http://www.servicos.capes.gov.br);

- Portal de Teses e Dissertações em Saúde Pública da Biblioteca Virtual de Saúde (BVS) (http://thesis.cict.fiocruz.br);

- Base de dados LILACS (Literatura Latino-Americana e do Caribe em Ciências da Saúde), também acessado por: http://bases. bireme.br, base de dados: tesessp;

- Base de dados Thesis;

- Base de dados BDTD (Biblioteca Digital de Teses e Dissertações), também acessado por http://bdtd.ibict.br/busca/avancada.jsp;

- VTLS (Virginia Technical Library System), sendo esta uma base de dados adotada pela UERJ, acessada apenas pela rede da própria universidade;

- UFRGS (http://sabix.ufrgs.br);

- UFSC (http://teses.eps.ufsc.br);

- PUC-SP (www.sapientia.pucsp.br);

- UNICAMP (http://liddigi.unicamp.br);

- UNESP (http://hours.cgb.unesp.br/cgb/biblioteca);

- USP (www.teses.usp.br);

- UFBA (www.odo.ufba.br/posgrad).

Foram utilizados os descritores: "política", "saúde pública", "saúde coletiva" e "SUS". Foi também utilizada a pesquisa do termo "Estado do Rio de Janeiro".

Os trabalhos encontrados foram classificados em oito áreas temáticas: Políticas; Epidemiologia; Recursos Humanos; Saúde e Sociedade; Formação Profissional; Modelos de Atenção e Gestão, Sistemas de Informação e História da Saúde Pública. Entretanto, para uma melhor sistematização dos dados da pesquisa, os autores julgaram conveniente dividir a categoria Políticas, onde foi encontrado um total de 22 trabalhos, em duas subcategorias: Políticas do SUS (Sistema Único de Saúde) e Políticas Especificas. A agregação dos trabalhos nessas categorias foi realizada pelos autores, calibrados para este fim, que tomaram por base a leitura e análise dos resumos disponibilizados nas bases de dados pesquisadas.

Considerando que alguns trabalhos, a despeito de trazerem em seu título uma menção ao Estado do Rio de Janeiro, referiram-se apenas a um ou alguns 
municípios dessa unidade da federação, esta pesquisa adotou um critério de inclusão. Assim, apenas foram contabilizados os trabalhos que estudaram o Estado do Rio de Janeiro em amostras superiores a 23 municípios, ou seja, superiores a $1 / 4$ do total dos 92 municípios do estado.

No tocante às áreas temáticas, os trabalhos classificados em Politicas do SUS foram aqueles que abordaram temas como: o processo de implantação do SUS, as temáticas do federalismo brasileiro, do processo de descentralização, as relaçōes intergovernamentais, as instâncias colegiadas, os consórcios institucionais de saúde, a garantia de acesso à atenção básica e a equidade no financiamento do SUS. Na subcategoria Políticas Específicas, foram contabilizados aqueles trabalhos que se voltaram para a Reforma Psiquiátrica, as diretrizes e normas nacionais de reabilitação, a Política Nacional do Idoso, a Política Nacional de Medicamentos Genéricos, o Programa Nacional de Alimentação Escolar (PNAE), a Política de Atenção Integral a Usuários de Álcool e Outras Drogas, o Projeto de Profissionalização dos Trabalhadores da Área de Enfermagem (PROFAE) e o Programa de Imunizações do Estado do Rio de Janeiro (PIERJ).

A categoria Epidemiologia incluiu apenas aqueles trabalhos que, utilizando-se de técnicas epidemiológicas de pesquisas, correlacionaram seus dados/resultados com os aspectos relacionados à qualidade de vida de indivíduos e populações.

A categoria Recursos Humanos incluiu aqueles trabalhos que verificaram a inserção de profissionais médicos no Programa de Saúde da Família e formação e prática do pessoal auxiliar.

Saúde e Sociedade foi o termo escolhido para a categorização daqueles trabalhos que se orientaram pela autopercepção da saúde, representações sociais, subjetividades e significados das condições de saúde, relacionando-as ao meio ambiente, trabalhos que lançam mão de questionários e/ou entrevistas para avaliações qualitativas sobre os serviços e a atenção à saúde e a percepção do trabalhador e do usuário sobre seus direitos à saúde.

Em Formação Profissional, foram classificados aqueles trabalhos que tiveram como objeto os currículos profissionais, os cursos de pós-graduação e de aperfeiçoamento profissional.

$\mathrm{Na}$ área temática Modelos de Atenção e Gestão, foram classificados aqueles trabalhos que abordaram as questôes relativas a assistência hospitalar, produção dos laboratórios de análises clínicas e oferta e produção dos serviços de atenção à saúde. 
Em Sistemas de Informação, os autores classificaram aqueles trabalhos voltados para a existência e a qualidade dos sistemas de informação em saúde.

Finalmente, em História da Saúde Pública, incluem-se aqueles trabalhos que tomaram por base a história das instituições e dos atores sociais que participaram da construção do sistema de saúde no âmbito do estado do Rio de Janeiro.

Além de categorizados por áreas temáticas, os trabalhos foram contabilizados segundo sua instituição de origem, ano de defesa e nível de mestrado ou doutorado.

\section{Resultados}

No Estado do Rio de Janeiro, de acordo com critérios desta pesquisa, no período de 1960 até 15 de julho de 2007, foram contabilizados 49 trabalhos. Entretanto, até 1990 foi encontrado apenas um trabalho com tais características. Os outros 48 trabalhos foram produzidos entre 1991 e 2007. Essa produção é composta por 38 dissertações de mestrado e 11 teses de doutoramento. Vale ressaltar que a presente pesquisa considerou os mestrados acadêmico e profissionalizante. No entanto, destacamos que, no total de trabalhos pesquisados, foram encontradas apenas duas dissertaçóes de mestrado profissionalizante, o que confirma a hegemonia dos cursos de mestrado acadêmico.

O gráfico 1 ilustra a distribuição de trabalhos entre dissertaçōes de mestrado e teses de doutoramento, evidenciando que há uma grande concentração em dissertações de mestrado.

\section{Gráfico 1}

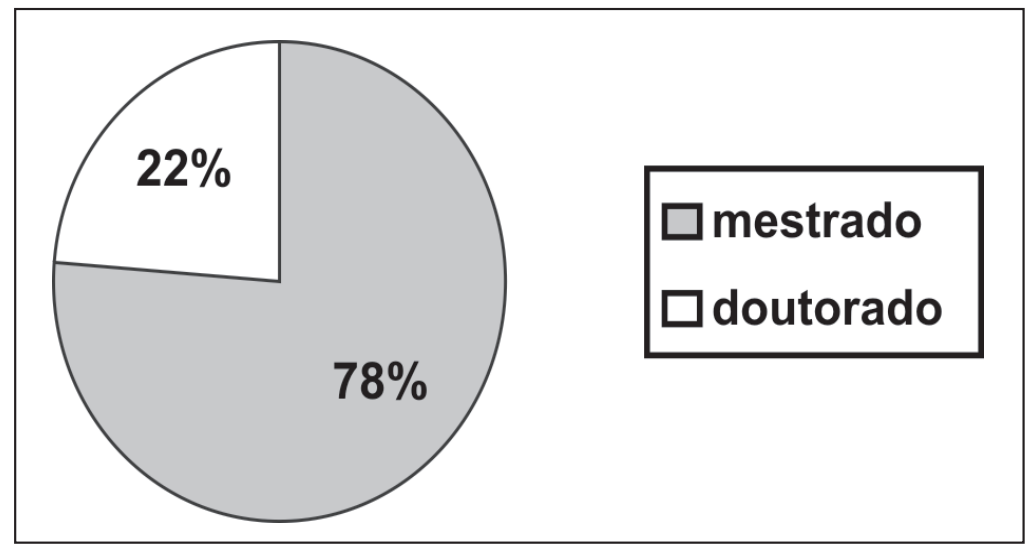

FONTE: elaboração própria 
No conjunto da produção pesquisada, foi encontrado somente um autor ${ }^{3}$ que realizou dois trabalhos sequenciados, ou seja, fez sua dissertação de mestrado e sua tese de doutoramento na mesma área de conhecimento: Políticas do SUS. Ademais, ambos os trabalhos foram produzidos na mesma instituição de ensino e pesquisa, e sob a mesma orientação acadêmica.

O gráfico 2 mostra a produção acadêmica no período total pesquisado. $\mathrm{O}$ período compreendido entre os anos de 1960 a 1990 foi omitido graficamente, uma vez que apenas um trabalho (OLIVEIRA, 1980) foi produzido pelo IMSUERJ. A pesquisa revelou que, no período compreendido entre 1960 e 2000, o volume máximo de produção anual foi de três títulos, obtido em 1996. Portanto, no período acima citado, o volume da produção acadêmica na área apresentou-se muito baixo, conforme ilustra o gráfico 2 .

No entanto, a partir de 2001, e sobretudo nos anos de 2003 e 2004, ocorreu notável expansão da produção, contabilizando um total de 29 trabalhos nesses quatro anos. Deste total, 12 trabalhos estiveram concentrados em 2003 e nove em 2004.

Nos anos subsequentes (2005 e 2006) observou-se uma tendência declinante, sendo que no ano de 2007 , até 15 de julho, nenhum trabalho foi encontrado de acordo com os critérios desta pesquisa. Configurou-se então uma carência de trabalhos no âmbito da Saúde Coletiva referentes ao Estado do Rio de Janeiro, cabendo ressaltar que os anos de 2003 e 2004 apresentaram-se como uma exceção.

\section{Gráfico 2}

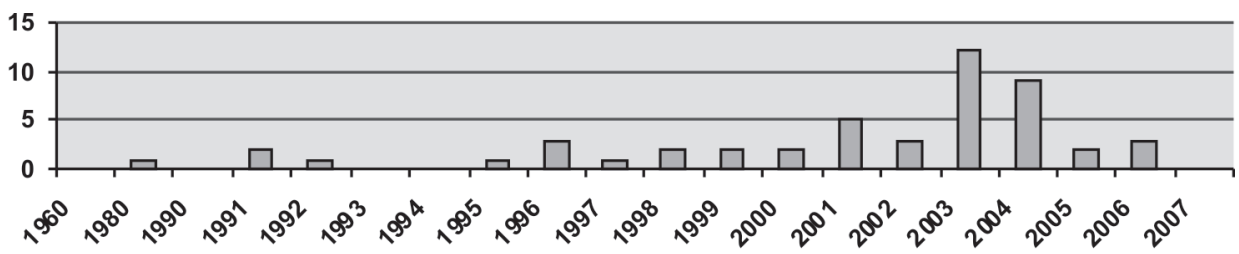

$\square$ produção anual

Fonte: elaboração própria.

No tocante à distribuição institucional dos trabalhos exposta no gráfico 3, a seguir, podemos verificar que a produção concentrou-se em apenas três instituições: Fundação Oswaldo Cruz (Fiocruz), ${ }_{4}^{4}$ IMS-UERJ e Universidade Federal do Rio de 
Janeiro (UFRJ). Vale registrar que a UFRJ, para fins desta pesquisa, compreende a Faculdade de Enfermagem e o Núcleo de Estudos em Saúde Coletiva (NESC). 5

Observando mais atentamente a produção, podemos afirmar que a Fiocruz, que realizou o maior volume de trabalhos, contribuiu com 21 dissertaçōes $^{6}$ de mestrado e quatro teses de doutoramento. O IMS/UERJ produziu 13 dissertaçôes de mestrado e sete teses de doutoramento. A UFRJ é responsável por quatro dissertações de mestrado. O gráfico 3, abaixo, ilustra a distribuição da produçãoo entre mestrado e doutorado por instituição.

\section{Gráfico 3}

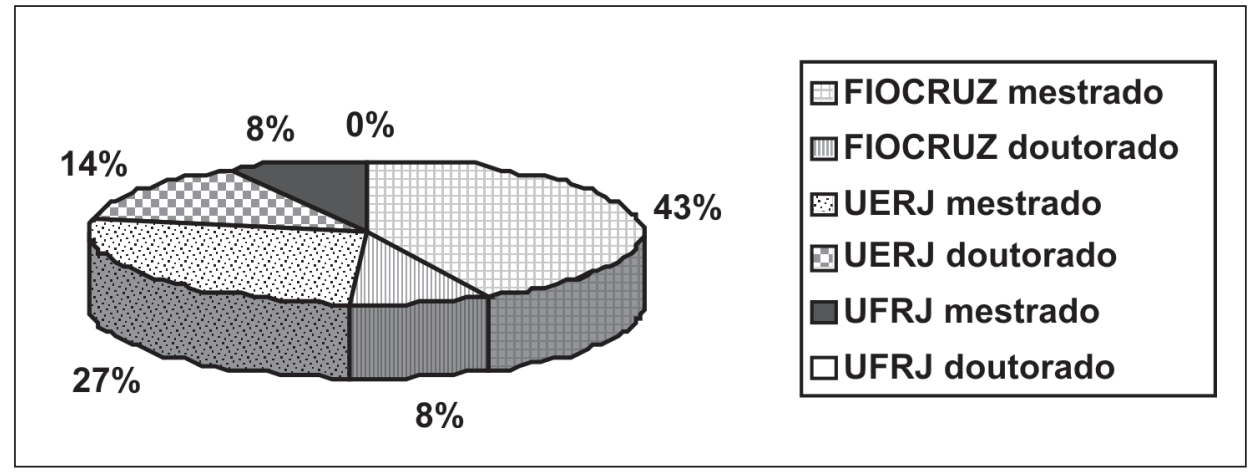

Fonte: elaboração própria.

Em relação às áreas temáticas, a pesquisa revelou que, do total de 49 trabalhos encontrados, 22 se incluem na categoria Políticas (45\%). Destes, 10 trabalhos (20\%) foram classificados na subcategoria Politicas do SUS e 12 trabalhos (25\%) na subcategoria Políticas Específicas. Portanto, a área temática Politicas, incluindo suas duas subcategorias, é a que detém o maior número de trabalhos.

Em Epidemiologia foram produzidos 11 trabalhos, o que corresponde a 23\% do total dos trabalhos pesquisados. Os restantes 32\% do total de trabalhos são de responsabilidade das demais áreas temáticas. As áreas de Recursos Humanos, Saúde Sociedade e Sistemas de Informação produziram cada uma 6\% do total de trabalhos. A área temática Modelos de Atenção e Gestão contabilizou 8\% dos trabalhos. Em Formação Profissional, foram contabilizados $4 \%$ dos trabalhos, enquanto que em História da Saúde Pública foram produzidos apenas $2 \%$ desses trabalhos.

O gráfico 4 ilustra a distribuição dos trabalhos por áreas temáticas. 


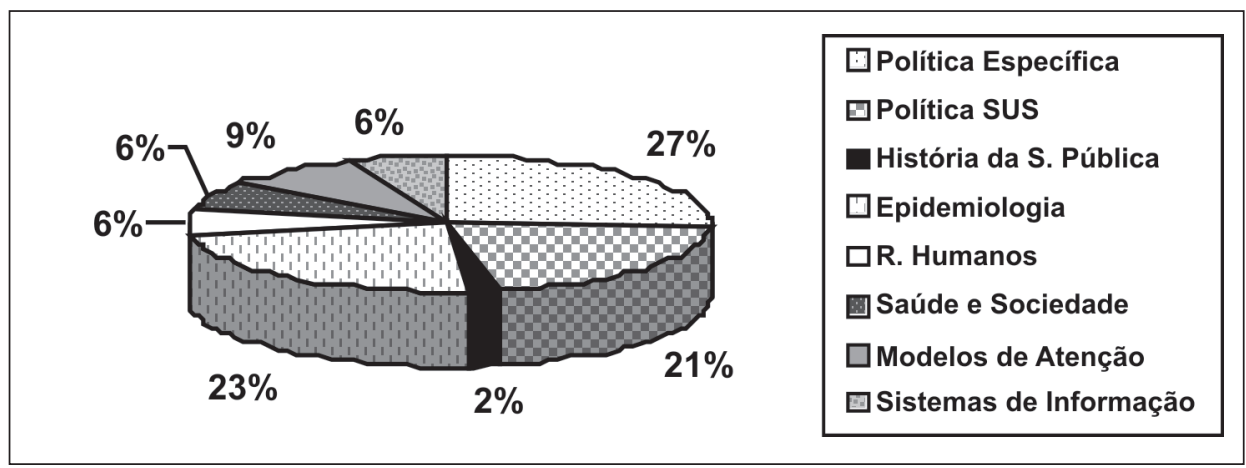

Fonte: elaboração própria.

\section{Discussão}

Considerando a história política da formação do Estado do Rio de Janeiro e suas consequentes implicações no contexto da saúde estadual, ${ }^{7}$ podemos concluir que a produção bibliográfica de dissertações de mestrado e teses de doutoramento sobre o Estado do Rio de Janeiro e seu sistema de saúde é ainda muito baixa e mal distribuída. Ademais, essa produção é incompatível com a importância econômica do estado, uma vez que o valor do seu Produto Interno Bruto (PIB) é o segundo maior do país (IJSN, 2008). Da mesma forma, é incompatível com a importância da sua condição sócio-política, expressa por sua população, que em 2007 foi estimada em 15.420.375 habitantes (IBGE, 2008).

A diferença do volume da produção de trabalhos nos níveis mestrado e doutorado é bastante significativa: as dissertaçôes de mestrado contabilizaram um número mais do que três vezes maior que a produção no nível de doutorado. Este fato aponta tanto para uma necessidade de expansão da pesquisa no nível de doutorado neste campo temático, como para a descontinuidade das titulaçôes no interior desta área temática.

A produção em sequência por área de conhecimento e sob a mesma orientação acadêmica ocorreu apenas uma vez no universo de trabalhos pesquisados, o que pode indicar que o interesse de pesquisa está mais fortemente relacionado a temas do que aos estudos relativos ao Estado do Rio de Janeiro e suas especificidades. Investigações futuras sobre esta questão poderão integrar uma agenda de pesquisa sobre esta unidade da federação. 
De um modo geral, o volume da produção acadêmica neste campo temático não é estável. A produção apresentou um pico, entre 2003 e 2004, retornando em seguida para o seu perfil de baixo volume. Vale registrar que este fato não é compatível nem com a complexidade dos assuntos relativos à Saúde Coletiva no âmbito do estado, nem com as diretrizes vigentes do SUS. ${ }^{8}$ Das três instituições que produziram trabalhos desse tipo, apenas a Fiocruz não possui cursos de graduação. No entanto, essa instituição apresentou o maior volume de produção: 51\% dos trabalhos. Nesse sentido, podemos observar também que a produção da UERJ é totalmente de responsabilidade do IMS, enquanto que a produção da UFRJ está dividida entre sua Faculdade de Enfermagem (apenas um trabalho) e o NESC/ IESC. É possível que a concentração da produção na Fiocruz e em núcleos de pósgraduação esteja relacionada ao baixo grau de interesse que este campo temático exerce nos níveis de graduação das instituições de nível superior fluminenses.

Vale notar que as instituições responsáveis por esta produção têm importância nacional no campo da saúde coletiva. Dessa forma, é surpreendente o baixo volume desta produção intelectual, quando referida ao estado do Rio de Janeiro.

Ainda no que diz respeito às instituições de ensino superior públicas, a Universidade Federal Fluminense (UFF, localizada no município de Niterói), apesar de contar com o Instituto de Saúde da Comunidade, criado em abril de 1997, somente em março de 2008 passou a oferecer o curso de mestrado em Saúde Coletiva, não tendo, portanto, contribuído com qualquer trabalho que atenda aos critérios desta pesquisa (UFF, 2009).

No tocante às universidades privadas, durante o período pesquisado, estas estiveram totalmente ausentes do escopo da presente pesquisa. No entanto, o curso de mestrado em Saúde da Família oferecido pela Universidade Estácio de Sá, iniciado em abril de 2005, ainda não gerou publicações capazes de serem indexadas nas bases de dados consultadas por esta pesquisa, uma vez que a primeira turma deste curso, a princípio, começou apresentar suas dissertações a partir de fevereiro de 2007.

Em relação à distribuição da produção por áreas temáticas, os dados apontam que há uma concentração de trabalhos voltados para estudos das Políticas (45\%). $\mathrm{Na}$ área de Epidemiologia, foram desenvolvidos 23\% dos trabalhos. A despeito da importância do estudo das políticas para a operacionalização do sistema de saúde como um todo, e dos estudos epidemiológicos para o desenvolvimento 
destas políticas sob a ótica territorial, é notável o fato de que as áreas Recursos Humanos, Saúde e Sociedade, Sistemas de Informação (6\% cada) e Modelos de Atenção e Gestão (8\%) tenham apresentado baixo volume de produção. Esta baixa densidade é incompatível com a extrema importância estratégica dessas quatro áreas temáticas para a sustentação do SUS no âmbito regional. Aqui a carência de estudos mostra a lacuna a ser mais prontamente superada.

\section{Considerações finais}

Em artigo intitulado "O SUS no Rio de Janeiro", e que foi publicado na revista Physis em 2001, Viana, Levcovitz e Dal Poz afirmam que:

[...] a alta heterogeneidade socioeconômica, política, cultural, demográfica e epidemiológica produz modelos próprios de saúde (regionais ou locais). Esses modelos, por sua vez, são identificados por um rol de características singulares que só podem ser apreendidas a partir de estudos dirigidos para o exame dessas mesmas realidades, uma vez que as configuraçôes locais são dependentes de inúmeras variáveis (VIANA; LEVCOVITZ; DAL POZ, 2001, p. 15-16).

Neste sentido, o processo de desenvolvimento do SUS e as diversas políticas específicas de saúde implantadas no Estado do Rio de Janeiro, somados à complexidade econômica e social fluminense, tornam necessária e urgente a expansão dos esforços de investigação acadêmica nos níveis de mestrado e doutorado, no sentido de subsidiar o planejamento, a gestão e a operacionalização do SUS no âmbito do estado.

Ademais, com as perspectivas futuras da expansão da produção de petróleo e gás envolvendo 11 municípios 9 que compõem o Consórcio Intermunicipal para o Desenvolvimento do Leste Fluminense (CONLESTE), haverá impactos positivos na economia. Esses impactos diretos e indiretos poderão repercutir positivamente tanto na oferta quanto na demanda por serviços sociais, nos quais se incluem os de saúde. No tocante à oferta, a ampliação da receita tributária direta e indireta ampliará a base de financiamento das políticas sociais em geral e da saúde em particular. No tocante à demanda, a ampliação e redirecionamento da base populacional dos municípios envolvidos neste processo de mudança socioeconômica deverão implicar uma reestruturação da assistência a saúde no estado. E a demanda oriunda da expansão da atividade produtiva voltada direta e indiretamente para a exploração do petróleo e gás, e da consequente organização do trabalho nestes 
setores da economia, demandará cada vez mais a elaboração de estudos e pesquisas voltados para a reorganização do SUS no âmbito desses municípios.

Neste contexto, a complexidade do estado em várias áreas, sobretudo no que diz respeito à saúde pública, justifica estímulos específicos para subsidiar pesquisas no âmbito do estado. A Fundação Carlos Chagas Filho de Amparo à Pesquisa do Estado do Rio de Janeiro (FAPERJ) mantém pelo menos três programas que contam com linhas de financiamento para o apoio a pesquisas voltadas para o âmbito do estado, a saber: Programa Pensa Rio, Pesquisa para o SUS (PP-SUS) e Programa de Parceria de Pós Graduação (PPPG). Esse tipo de incentivo deve servir de exemplo e estímulo para empresas públicas e privadas com interesse econômico na região subsidiarem, da mesma forma, as pesquisas acadêmicas voltadas para este campo da saúde.

A despeito desses progressos no âmbito da oferta de programas de financiamento à pesquisa, acreditamos que qualquer ampliação significativa no volume de dissertaçóes de mestrado e teses de doutoramento voltadas para um maior conhecimento acerca do sistema de saúde no Estado do Rio de Janeiro passará necessariamente por uma estratégia centrada nos cursos de graduação, não apenas na área biomédica, mas também na área de ciências humanas e sociais. Essa estratégia deverá sensibilizar cursos multidisciplinares, bolsas de iniciação científica e monografias de final de curso de estudantes graduandos, que irão direcionar seus esforços acadêmicos no sentido de contribuir para um maior conhecimento da realidade que se faz presente no campo da Saúde Coletiva no âmbito do Estado do Rio de Janeiro.

Vale enfatizar que essa necessidade de sensibilização não deverá estar restrita à graduação. Os graduandos de diversas formações profissionais deverão ser incentivados a ingressar em programas de mestrado, doutorado e outros programas de pós-graduação tendo em vista a necessidade de desenvolvimento de temas relativos ao Estado do Rio de Janeiro. Nesta perspectiva, o foco na pós-graduação deve privilegiar os estudos sequenciais de mestrado e doutorado em temas afeitos ao campo da saúde coletiva nesta unidade da federação. Ademais, estes estudos devem estar orientados para aqueles campos temáticos de menor densidade e maior importância estratégica para o desenvolvimento do sistema de saúde no estado.

As três instituições de pesquisa responsáveis por esta produção intelectual (Fiocruz, IMS-UERJ e IESC-UFRJ) não devem descartar a possibilidade de 
estabelecer cooperações interinstitucionais orientadas para superar as lacunas identificadas por esta pesquisa. Essa cooperação poderá se estender para outras instituições públicas ou privadas com interesse no campo da saúde coletiva.

Por fim, cabe ressaltar que a união dos esforços entre as instituiçōes acadêmicas e os atores envolvidos no processo de desenvolvimento do sistema de atenção à saúde neste estado poderá tornar factível a superação dos desafios tão claramente associados à melhoria das condições de vida da população fluminense, especialmente daquelas afeitas ao campo da saúde.

\section{Referências}

BRASIL. Ministério da Saúde. Lei n. 8080, de 19 de setembro de 1990. Dispõe sobre as condiçôes para a promoção, proteção e recuperação da saúde; a organização e o funcionamento dos serviços correspondentes e dá outras providências. Disponível em: <http://www6. senado.gov.br/sicon $/$ ListaReferencias.action? codigoBase $=2$ \&codigoDocumento $=134561>$ Acesso em: 02 mar. 2007.

. Portaria GM/MS no 399, de 22 de fevereiro de 2006. Divulga o Pacto pela Saúde 2006 - Consolidação do SUS e aprova as Diretrizes Operacionais do Referido Pacto. Disponível em: <http://dtr2001.saude.gov.br/sas/PORTARIAS/Port2006/GM/GM-399. htm> Acesso em: 20 fev. 2007.

BIBLIOTECA VIRTUAL EM SAÚDE. Descritores em Ciências da Saúde (DeCS). Termo Saúde Bucal, 2007. BIREME/OPAS /OMS. Disponível em: <http://decs.bvs.br/cgibin/wxis1660. exe/decsserver/?IsisScript=../cgibin/decsserver/decsserver.xis \&task=exact_term \&previous_ page $=$ homepage $\&$ interface_language $=$ p \&search_language $=p \&$ search_exp $=$ Saúde\%20 Bucal\&show_tree_number=T> Acesso em: 02 mar. 2007.

DAVIDOVICH, F.R. Estado do Rio de Janeiro: singularidades de um contexto territorial. Revista Território. Rio de Janeiro, n. 9, p. 9-24. jul/dez 2000 . Disponível em: <http://146.164.23.131/pdf/N_09/v-9-davidovich.pdf> Acesso em: 02 mar. 2007.

INSTITUTO BRASILEIRO DE GEOGRAFIA E ESTATÍSTICA. Contas Nacionais, $\mathrm{n}^{\circ}$ 25. Contas Regionais do Brasil 2003-2006. Rio de Janeiro, 2008. Disponível em: <http:// www.ibge.gov.br/home/estatistica/economia/contasregionais/2003_2006/contasregionais2003_2006.pdf> Acesso em: 10 abr. 2009.

INSTITUTO JONES DOS SANTOS NEVES. Disponível em <http://www.ijsn.es.gov. br/contasregionais/tabelas08/Ranking\%20das\%20UFs\%20no\%20PIB.xls>. Acesso em: dez. 2008.

KORNIS, G.E.M.; MAIA, L.S.; FORTUNA, R.F.P. A produção intelectual em Saúde Bucal na perspectiva coletiva: uma análise da experiência brasileira de 1990 a 2006. Rio de Janeiro: IMS-UERJ, 2006 (Série Estudos em Saúde Coletiva, 222). 
LIMA, L.D. A Comissão Intergestores Bipartite a CIB do Rio de Janeiro. PHYSIS. Rev. de Saúde Coletiva. Rio de Janeiro, v. 11, n. 1, p. 199-252, 2001

MAIA, L.S. Política Nacional de Saúde Bucal: uma análise da reorganização da atenção à saúde bucal no estado do Rio de Janeiro frente aos incentivos federais. Dissertação (Mestrado em Saúde Coletiva) - Instituto de Medicina Social, Universidade do Estado do Rio de Janeiro, Rio de Janeiro, 2008 213p.

OLIVEIRA, J. de A. Trabalho médico no Rio de Janeiro. Dissertação (Mestrado em Medicina Social) - Instituto de Medicina Social, Universidade do Estado do Rio de Janeiro, Rio de Janeiro, 1980.

PARADA, R.: Sistema Estadual de Saúde. Descentralização e relações intergovernamentais: um estudo sobre o Estado do Rio de Janeiro. Dissertação (Mestrado em Saúde Coletiva) Instituto de Medicina Social, Universidade do Estado do Rio de Janeiro, Rio de Janeiro, 1997. $202 \mathrm{p}$.

PARADA, R. Federalismo e o SUS: um estudo sobre as mudanças dos padrões de governabilidade no Rio de Janeiro. Tese (Doutorado em Saúde Coletiva) - Instituto de Medicina Social, Universidade do Estado do Rio de Janeiro, Rio de Janeiro, 2002 156p.

SANTOS, A.M.S.P. Economia, espaço e sociedade no Rio de Janeiro. Rio de Janeiro: FGV, 2003.

UNIVERSIDADE FEDERAL FLUMINENSE. Plano de Expansão e Reestruturação: Proposta Detalhada Instituto de Saúde da Comunidade. Projeto Acadêmico Institucional. Disponível em: www.uff.br/pdi/images/pdf/expansao-projetos/Saude-da-comunidade.pdfAcesso em: 02 maio 2009.

VALLADARES, L. do P.; SANT'ANNA M.J.G. O Rio de Janeiro em Teses: Catálogo Bibliográfico 1960-1990. Rio de Janeiro: Urbandata/CEP:Rio-UERJ, 1992.

VIANA, A.L.; LEVCOVITZ, E.; DAL POZ, M. O SUS no Estado do Rio de Janeiro. PHYSIS. Rev. de Saúde Coletiva. Rio de Janeiro, v. 11, n. 1, p. 11-17, 2001

\section{Notas}

${ }^{1}$ A produção intelectual total no período compreendido entre 1960 e 1990 apresentou uma média anual de 6,6 trabalhos/ano. A produção intelectual em Saúde Coletiva para este mesmo período foi de apenas 0,2 trabalhos/ano.

${ }^{2}$ As estruturas das bases de dados consultadas para a realização desta pesquisa estão em processo de modificações nos seus instrumentos de busca desde a realização do $8^{\circ}$ Congresso Regional de Informação em Ciências da Saúde (CRICS), ocorrido em setembro de 2008. Nesse sentido, estas alterações inviabilizam uma busca atual utilizando o mesmo caminho metodológico.

${ }^{3}$ Roberto Parada, sob orientação da Prof. Dra Ana Luiza D’Avila Viana, no IMS-UERJ (PARADA, 1997 e 2002).

${ }^{4}$ A Fiocruz, para fins desta pesquisa, também compreende a Escola Nacional de Saúde Pública Sergio Arouca (ENSP). 
${ }^{5}$ Em 14 de setembro de 2006, o Núcleo de Estudos em Saúde Coletiva (NESC) tornou-se o Instituto de Estudos em Saúde Coletiva (IESC).

${ }^{6}$ Destacamos que, dentre as dissertaçôes de mestrado desenvolvidas pela Fiocruz, apenas duas foram produzidas por curso de mestrado profissionalizante na ENSP.

7 Segundo Parada (1997), a história política e sanitária do Estado do Rio de Janeiro, somada às dificuldades inerentes ao próprio processo de implantação do SUS, acabaram por imprimir muitas especificidades, não só ao sistema de saúde estadual como também aos sistemas municipais do estado. As consequências dessas especificidades ainda se traduzem como problemáticas na atualidade.

${ }^{8}$ A Lei no 8080/90, no capítulo que trata de Competências e Atribuições do SUS, destaca em seu artigo 15, item XIX, a importância da realização de pesquisas e estudos na área da saúde. Somam-se a isto as diretrizes de regionalização contidas no Pacto Pela Saúde de 2006. Para tal, o conhecimento aprofundado do estado e suas regiôes se tornam fundamentais para a operacionalização do SUS.

${ }^{9}$ Rio Bonito, Maricá, Itaboraí, Magé, Niterói, São Gonçalo, Tanguá, Silva Jardim, Cachoeiras de Macacu, Guapimirim e Casemiro de Abreu. 
The intellectual production in Public Health on Rio de Janeiro State, Brazil: from 1960 to 2007 This text focuses on the intellectual production in public health studies expressed through master's degree dissertations and doctoral thesis whose theme was Rio de Janeiro State, Brazil. The research considered the period from 1960 to July $15^{\text {th }}, 2007$. From 1960 to 1990 there was only one work on the Rio de Janeiro State, and from 1991 to July $15^{\text {th }}, 2007$, we found 48 works on public health studies concerning Rio de Janeiro State. The intellectual production was classified in eight thematic areas: Policies; Epidemiology; Human Resources; Professional Education and Training; Health and Society; Assistance Models and Management; Information Systems and Public Health History. The theme area named Policies is the production leader with 22 works (and it was divided in two: National Health System Policies (20\%) and Specific Health Policies (20\%). The theme area named Epidemiology, with 11 works (23\%), is another important concentration pole. The intellectual production as a whole had been concentrated both in master's degree dissertations (78\%) as in a few number of educational and research institutions such as Fiocruz - the leader in terms of master's degree dissertations and IMS-UERJ, which holds the leadership in terms of doctoral thesis. In spite of the great importance of Rio de Janeiro State in relation to Brazil, we conclude that the intellectual production is reduced and concentrated in a few theme areas.

$>$ Key words: Intellectual Production, Public Heath, Rio de Janeiro State. 